\title{
Zuverlässige numerische Analyse linearer Regelungssysteme
}

Von Dr.-Ing. Ferdinand Svaricek

ITT Automotive Europe GmbH, Frankfurt/Main 
Vom Fachbereich Maschinenbau der Gerhard-Mercator-Universität - GH Duisburg genehmigte Habilitationsschrift (Datum der Feststellung der Lehrbefähigung: 4. Februar 1994)

Die Deutsche Bibliothek - CIP-Einheitsaufnahme

\section{Svaricek, Ferdinand :}

Zuverlässige numerische Analyse linearer Regelungssysteme /

von Ferdinand Svaricek. - Stuttgart : Teubner, 1995

ISBN 978-3-519-06175-5

ISBN 978-3-322-90142-2 (eBook)

DOI 10.1007/978-3-322-90142-2

Das Werk einschließlich aller seiner Teile ist urheberrechtlich geschützt. Jede Verwertung außerhalb der engen Grenzen des Urheberrechtsgesetzes ist ohne Zustimmung des Verlages unzulässig und strafbar. Das gilt besonders für Vervielfältigungen, Übersetzungen, Mikroverfilmungen und die Einspeicherung und Verarbeitung in elektronischen Systemen.

(c) B. G. Teubner Stuttgart 1995

Umschlaggestaltung: Peter Pfitz, Stuttgart 


\section{Vorwort}

Diese Arbeit setzt sich mit der zuverlässigen numerischen Ermittlung grundlegender Eigenschaften von Regelungssystemen auseinander, die hinreichend genau durch ein lineares Modell, das lediglich eine Näherung 1. Ordnung darstellt (Schwarz 1991), approximiert werden können. Neben der Steuerund Beobachtbarkeit stehen Eigenschaften wie die Invertierbarkeit, die Ein/Ausgangsentkoppelbarkeit, die Störentkoppelbarkeit und das Verhalten bei hohen Rückführverstärkungen im Mittelpunkt des Interesses. Alle diese Eigenschaften sind im Grunde mit entsprechend definierten Nullstellen des Systems eng verknüpft. Einen breiten Raum wird daher der Behandlung des Konzeptes der endlichen und unendlichen Nullstellen von Mehrgrößensystemen eingeräumt.

An einem Modell niedriger Ordnung eines Werkzeugmaschinenantriebes wird zunächst demonstriert, wie stark numerisch ermittelte Aussagen durch die begrenzte Rechengenauigkeit der verwendeten Gleitpunktarithmetik beeinflußt werden können. Anschließend werden dann die bekannten Kriterien zur Überprüfung der Steuerbarkeit auf ihre numerischen Eigenschaften hin untersucht. Ein Fazit dieser Untersuchung ist, daß alle Kriterien bei größeren Systemen und einer numerischen Auswertung mit einer begrenzten Anzahl von Dezimalstellen völlig falsche Ergebnisse liefern können, so daß die mit konventionellen Programmen gewonnenen Aussagen stets als "fragwürdig“ angesehen werden müssen.

Aufgrund der begrenzten Rechengenauigkeit der verfügbaren Computer haben konventionelle Programme, die ein meist noch nicht einmal exakt bekanntes quantitatives Modell auswerten, gerade bei der Beantwortung qualitativer Fragen, wie z.B.: „Ist ein System vollständig steuerbar oder nicht?" oder „Besitzt ein System Nullstellen oder nicht?" besondere Schwierigkeiten. In diesem Zusammenhang ist folgende Erkenntnis von elementarer Bedeutung: Mit Hilfe von Programmen, die eine Gleitpunktarithmetik mit endlicher Genauigkeit verwenden, kann die Frage nach der Steuerbarkeit eines Systems nicht eindeutig mit Ja oder Nein beantwortet werden, da die Steuerbarkeit ihrem Wesen nach eine strukturelle Eigenschaft ist, und jedes nicht steuerbare System $(\mathbf{A}, \mathbf{B})$ beliebig nahe an einem steuerbaren System $(\mathbf{A}+\delta \mathbf{A}, \mathbf{B}+\delta \mathbf{B})$ liegt. Durch die im Rahmen einer numerischen Überprüfung auftretenden Rundungsfehler wird ein nicht steuerbares System dann derart gestört, daß es wieder steuerbar wird. Aus numerischer Sicht kann eigentlich nur die Frage "Wie weit ist ein steuerbares System vom nächsten nicht steuerbaren System entfernt?“ zuverlässig beantwortet werden.

Fragt man bei einem nicht steuerbaren System nach den zugrundeliegenden Ursachen, so können zwei Fälle unterschieden werden:

i) Der Verlust der Steuerbarkeit wird durch eine ungünstige Kombination der Zahlenwerte der Modellparameter hervorgerufen. Werden diese Werte 
allerdings nur leicht variiert, so erhält das System seine Steuerbarkeitseigenschaft zurück.

ii) Eine ungünstige Wahl der Stelleingriffe und/oder ein strukturelles Defizit im inneren Aufbau des Systems sind dafür verantwortlich, daß das System nicht steuerbar ist. Eine Variation der Zahlenwerte der Systemparameter reicht dann nicht aus um die Steuerbarkeit des Systems wieder herzustellen.

Der erste Fall kann aus praktischer Sicht als pathologisch angesehen werden, weil die Modellparameter meist weder exakt bekannt noch unveränderlich sind. Der Fall, daß ein System aufgrund der unter ii) angesprochenen Gründe nicht steuerbar ist, stellt also den auch für die Praxis relevanten Fall dar, dessen Auftreten nun mit Hilfe parameterunabhängiger Kriterien absolut zuverlässig erkannt werden kann. Bei rechnergestützten Analyse von Regelungssystemen sollte daher immer das in dieser Arbeit vorgestellte zweistufige Konzept angewendet werden:

i) In einem ersten Schritt werden zunächst nur einfache qualitative Strukturmodelle betrachtet. Anhand dieser Modelle wird dann mit Hilfe absolut zuverlässiger Programme, die lediglich ganzzahlige oder Boolesche Rechenoperationen einsetzen, untersucht, ob ein System z.B. aufgrund der gegebenen Struktur überhaupt steuerbar sein kann.

ii) Nur wenn diese Frage im ersten Schritt mit Ja zu beantwortet ist, werden anschließend die Eigenschaften des konkreten Zahlenmodells mit Hilfe von Programmen bestimmt, die aus numerischer Sicht besonders effizient und zuverlässig sind.

Eine solche Vorgehensweise ist, wie in dieser Arbeit dargestellt, nicht nur bei der Steuer- und Beobachtbarkeitsanalyse, sondern bei allen zuvor angesprochenen Analysefragen möglich. Dies ist auf die Tatsache zurückzuführen, daß die für diese Probleme maßgebliche Nullstellenstruktur im Unendlichen bereits mittels einfacher Strukturmodelle vollständig beschreibbar ist. So sind auch die in dieser Schrift vorgestellten Algorithmen zur Berechnung der generischen Nullstellenstruktur im Unendlichen, die lediglich die Kenntnis eines Strukturmodells voraussetzen, für eine Reihe regelungstechnischer Fragestellungen von grundlegender Bedeutung.

Die einzelnen Kapitel sind so gegliedert, daß nach einer Darstellung der theoretischen Grundlagen der behandelten Fragestellung die wichtigsten konventionellen Methoden und Algorithmen zur Beantwortung der Fragestellung einer kritischen Betrachtung und Wertung unterzogen werden. Zum Abschluß wird dann jeweils untersucht, inwieweit und mit welchen Verfahren zuverlässige parameterunabhängige Aussagen mit Hilfe eines Computers getroffen werden können. Die in diesem Buch empfohlenden Algorithmen und Programme sind entweder bereits in bekannten Programmsammlungen enthalten oder über Internet verfügbar. 
Bedingt durch die grundlegende Bedeutung der in diesem Buch angesprochenen Probleme, wendet sich dieses Buch nicht nur an Entwickler regelungstechnischer Software, sondern auch an diejenigen, die häufig Standardwerkzeuge, wie z.B. MATLAB oder Matrix/X, einsetzen und deren Grenzen kennenlernen wollen.

Dieses Buch ist die leicht überarbeitete Fassung meiner Habilitationsschrift „Zur rechnergestützten Analyse linearer Regelungssysteme", die vom Fachbereich Maschinenbau der Gerhard-Mercator-Universität - GH Duisburg als schriftliche Habilitationsleistung angenommen wurde. Dem Leiter des Fachgebietes Meß-, Steuer- und Regelungstechnik, Herrn Professor Dr.-Ing. H. Schwarz, gilt mein besonderer Dank für seine großzügige Unterstützung, seine Anregungen sowie für sein Interesse an dieser Arbeit. Mein Dank gilt ebenso den anderen Gutachtern für das Habilitationsverfahren, Herrn Prof. Dr. M. Braun und Herrn Prof. Dr. H. Kiendl, für ihr Interesse und ihre Unterstützung.

Danken möchte ich auch allen meinen ehemaligen Kolleginnen und Kollegen im Fachgebiet Meß-, Steuer- und Regelungstechnik, insbesondere Herrn Priv. Doz. Dr.-Ing. H.-D. Wend, für die stets vorhandene Diskussions- und Hilfsbereitschaft. Dem Verlag Teubner danke ich für die gute und verständnisvolle Zusammenarbeit.

Schließen möchte ich dieses Vorwort mit einem ganz besonders herzlichen Dank an meine Frau Gabriele, die mit viel Verständnis und Rücksichtnahme diese Arbeit ermöglicht hat. 


\section{Inhaltsverzeichnis}

1 Einleitung 1

2 Mathematische Grundlagen 9

2.1 Die Rosenbrock-Systemmatrix . . . . . . . . . . . . . . . 9

2.2 Rang einer Matrix . . . . . . . . . . . . . . . 10

2.2.1 Rang von Matrizenprodukten ............. 11

2.2 .2 Term-Rang und generischer Rang . . . . . . . . . . . . 12

2.3 Smithsche Normalform einer Polynommatrix . . . . . . . . . . . 14

2.4 Smith-McMillan-Normalform einer rationalen Matrix . . . . . . 16

2.5 Householder-Transformationen . . . . . . . . . . . . . . 19

2.6 Hessenberg-Form einer Matrix . . . . . . . . . . . . . . . 22

2.7 Singulärwertzerlegung und Anwendungen . . . . . . . . . . 23

2.7.1 Norm und Konditionszahl einer Matrix . . . . . . . . . . 24

2.7.2 Pseudoinverse einer Matrix . . . . . . . . . . 26

2.7.3 Numerische Berechnung der Singulärwertzerlegung . . . . . 28

2.7.4 Numerischer Rang einer Matrix . . . . . . . . . . . 29

2.8 Kronecker-Normalform eines Matrizenbüschels . . . . . . . . . . 29

2.8.1 Reguläre Matrizenbüschel . . . . . . . . . . . . . . 30

2.8 .2 Singuläre Matrizenbüschel . . . . . . . . . . . 33

2.8.3 Numerische Bestimmung der Kronecker-Normalform . . . 36

3 Steuerbarkeit linearer Regelungssysteme $\quad 38$

3.1 Steuerbarkeitskriterien . . . . . . . . . . . . . . . . . 40

3.1.1 Zustandssteuerbarkeit . . . . . . . . . . . . . 40

3.1 .2 Ausgangssteuerbarkeit . . . . . . . . . . . . . . 43

3.2 Quantitative Steuerbarkeitsanalyse . . . . . . . . . . . . 46

3.2.1 Steuerbarkeitsindizes . . . . . . . . . . . . . . 46 46

3.2.2 Abstand zum nächsten nicht steuerbaren System . . . . . 49

3.2 .3 Steuerbarkeitsmaße . . . . . . . . . . . . . 51

3.3 Numerische Untersuchung der Zustandssteuerbarkeit . . . . . . . . 59

3.3.1 Kalman-Kriterium . . . . . . . . . . . . . . 60

3.3 .2 Hautus-Kriterium ... . . . . . . . . . . . 71

3.3.3 Rosenbrocks Eingangs-Entkopplungsnullstellen . . . . . . 72

3.4 Qualitative Überprüfung der Zustandssteuerbarkeit . . . . . . . . 77

3.4.1 Strukturelle Steuerbarkeit . . . . . . . . . . . 78

3.4.2 Strenge strukturelle Steuerbarkeit . . . . . . . . . . 86

3.5 Numerische Untersuchung der Ausgangssteuerbarkeit . . . . . . . 92

3.5.1 Parameterabhängige Kriterien . . . . . . . . . . . 92

3.5.2 Parameterunabhängige Kriterien . . . . . . . . . . . . 94 
4 Beobachtbarkeit linearer Regelungssysteme $\quad 97$

4.1 Dualitätsprinzip . . . . . . . . . . . . . . . . 97

4.2 Kriterien der Beobachtbarkeit . . . . . . . . . . . 98

5 Pole und Nullstellen linearer Mehrgrößensysteme 102

5.1 Pole und Nullstellen der Übertragungsmatrix . . . . . . . . . . . . 103

5.2 Pole und Nullstellen der Rosenbrock-Systemmatrix . . . . . . . . 107

5.3 Eigenschaften der endlichen Nullstellen . . . . . . . . . . . . 116

5.4 Anzahl der endlichen Nullstellen eines linearen Systems . . . . . . 119

5.5 Physikalische Interpretation der Pole und Nullstellen . . . . . . 123

5.6 Praktische Bedeutung der endlichen Nullstellen . . . . . . . . . 126

5.7 Numerische Berechnung der endlichen Nullstellen . . . . . . . . . 127

5.7.1 Verfahren von Davison und Wang . . . . . . . . . . . 128

5.7 .2 Verfahren von $\mathrm{L}$ :ub und Moore . . . . . . . . . . . 129

5.7.3 Algorithmus ZEROS von Emami-Naeini und Van Dooren 133

5.7.4 Parameterunabhängige Abschätzung der Anzahl der Nullstellen . . . . . . . . . . . . . . . 142

5.7.5 Berechnung für Großsysteme . . . . . . . . . . . . 146

6 Nullstellen linearer Systeme im Unendlichen 153

6.1 Nullstellen im Unendlichen der Übertragungsmatrix . . . . . . . . . 154

6.2 Nullstellen im Unendlichen der Rosenbrock-Matrix . . . . . . . . . 155

6.3 Eine abstrakte allgemeingültigere Definition . . . . . . . . . . 159

6.4 Beziehungen zwischen den Nullstellendefinitionen im Unendlichen 162

6.5 Bestimmung der Nullstellenstruktur im Unendlichen . . . . . . . . 165

6.5.1 Bestimmung mit Hilfe der Bewertungstheorie . . . . . . . 166

6.5.2 Bestimmung anhand der Smith-McMillan-Form im Unendlichen ................... . . . . . . . . . .

6.5.3 Bestimmung mittels Toeplitz-Matrizen . . . . . . . . . 174

6.6 Eigenschaften der Nullstellen im Unendlichen . . . . . . . . . . . . . 177

6.7 Anzahl der Nullstellen im Unendlichen . . . . . . . . . . . . . . . 182

6.8 Praktische Bedeutung der Nullstellen im Unendlichen . . . . . . . 183

6.8.1 Ein-/Ausgangsentkopplung durch Zustandsrückführung . . 184

6.8.2 Das exakte dynamische Modellfolgeproblem . . . . . . . . 190

6.8.3 Das Störungs-Entkopplungsproblem . . . . . . . . . . . . . 192

6.8.4 Das Wurzelortskurvenverfahren für Mehrgrößensysteme . . 195

6.9 Numerische Berechnung der Nullstellen im Unendlichen . . . . . . 199

6.9.1 Der erweiterte Álgorithmus ZEROS . . . . . . . . . . . 201

6.10 Bestimmung der generischen Struktur im Unendlichen . . . . . . 206

6.10.1 Bestimmung mit Hilfe der Bewertungstheorie . . . . . . . 206

6.10 .2 Bestimmung mittels Toeplitz-Matrizen . . . . . . . . . . 211 
8 Literatır 219

Wichtige Formelzeichen und Abkürzungen 238

Algorithmenverzeichnis 241

Stichwortverzeichnis 242 